weakness in this direction, and our non-appreciation of and unwiliingness to perform our whole duty.

DISCUSSION.

Dr. Sennott, of Ohio, said he was interested in the treatment of diseases of children, on account of the action of medicines being untrammeled by mental predispositions.

Dr. Ulrich said children were shamefully abused by over-feeding and under-clothing, and ignorance regarding temperature, etc. He would hail with joy a revolution in the dress of the newly born; make the new dress of woolen material, loose, easily applied, etc.; would banish overlong skirts, pins, and belly bands. The dress now used for infants in this country was a torture, and the sooner a commonsense dress was adopted the better for future generations.

Dr. Boothby, of Wisconsin, agreed with Dr. Ulrich in the matter of dress, but would go further back in urging a revolution, back to the first bath, before any dress was used except a blanket. He would not allow a child to be bathed till from six to twelve hours had elapsed from its birth; would have the child thoroughly oiled, wrapped in soft, warm flannel, and laid by the side of the mother, and would not have mother or child disturbed for from a quarter to a half day; had seen fatal results from carelessness in bathing a weak babe in a cool room, and wrapping in cotton cloth; was particular to see that the babe was wrapped in flannel previously ziarmed und dried, and if because of circumstances it cannot be laid with the mother, keep near a warm stove.

Dr. Casebeer said, in closing the discussion, that what the profession needed in the medication of children, was pure and reliable medicine, given in water when possible, well diluted, small doses often repeated being generally preferable, and where this was followed by good judgment in other minutiæ, the regular physician would be preferred and employed many times where now the Homœopath is called.

\section{UNITY OF DIPHTHERITIC AND MEMBRANOUS CROUP,}

BY ALEX. HARRIS, M.D., VIRGINIA.

[Read before the Section on Diseases of Children, June, r 883.]

Because of the diverse views held by the profession at large, and especially the members of the district society which I have the honor to represent in part in this Association, I feel great interest in the discussion of the question, are Diphtheritic and Membranous Croup-the "Cynauche Trachialis of Cullen," the "True Croup" of other nosologists-convertible terms, modified only by difference in local expression, or are they distinct diseases, requiring opposite modes of treatment?

In the hope, then, of eliciting a discussion of this subject, which may at least secure a uniform therapy, I will assume the affirmative. Ours does not claim to be an exact, but a progressive science. At one time vensection was regarded the "sheet anchor" in the treatment of Asiatic cholera. Some of us have cured pneumonia-the cases amenable to the vis med- icince of that day-by " blood-letting to deliquium animi," tart-ant in one-half grain doses, ptyalism, and the absolute diet; and, if I mistake not, the estimated mortality under that treatment was one in three. Think of the host of victims to apoplexy which large bleedings and active catharsis did not cure! Some of us remember the frequent blood-lettings, the close room and light diet treatment of phthisis pulmonalis, and the "galloping consumption" of that day. I need but mention them, the vastly reduced mortality in all these diseases secured by an opposite therapy, to illustrate the advance made in therapeutics, May it not be claimed that the facilities for, and certainty of, diagnosis have advanced pari passu? How many of us remember when cough, quick pulse, with emaciation were the diagnostics of phthisis pulmonalis ; the "stitch in the side," pleurisy; brick dust expectoration, pneumonia, etc. Lately we depended upon general symptoms and physical signs to diagnose membranous laryngitis. Now we have the laryngoscope and microscope.

With this introduction I proceed to the consideration of the history of Membranous or Diptheritic Croup.

Diptheritic croup dates back certainly to the time of Areteus, if not, to that of Hippocrates.

The former of these writers, after describing pharyngeal diphtheria (as quoted by Dr. McKenzie), says: "If it extends rapidly to the chest through the windpipe, the patient dies the same day of suffocation." Galen refers to the expectoration of a "membranous tunic from the pharynx." But according to Dr. McKenzie, Baillou, who lived in the latter half of the sixteenth century, gives the first definite description of false membrane. Tilla Real, a Spanish physician, describes an epidemic which prevailed in I6II, in which you have "a white matter in the fauces, gullet and throat, of such a nature that if you stretch it with your hands it appears elastic, and has properties like those of wet leather."

Dr. House, of Edinburg, in 1765 described, under the name of croup, " an acute affection of the larynx and trachea, attended by the formation of a membrane in the pharynx, and often causing death by suffocation." Most of us, I presume, recognize diphtheria in this assemblage of symptoms.

In 1802 Dr. Cullen, of the same city, gave a description of cynanchi trachealis, in which we cannot fail to recognize the diphtheria of to-day; and Dr. Caldwell remarks, in a foot note, "that croup sometimes prevails epidemically, as appears to have been the case in the neighborhood of Alexandria, Va., in I 799, when Gen. Washington fell a victim to it.'

Pathologically, I think it may be assumed that false membrane is as constant a result of diphtheritic inflammation or poisoning, as the eruption in scarlatina, or pustule in small-pox is an effect of the poison upon which those diseases depend; and if this membrane is found in the pharynx, I think I am safe in saying that we all consider it equally conclusive as a diagnostic. It appears, then, only necessary to establish the identity of laryngeal with pharyngeal false membrane, to make the former as conclusive a diagnostic as the latter. 
I presume no one will undertake to differentiate laryngeal from pharyngeal false memhrane by the "naked eye" appearances. Virchow at one time based a distinction upon the claim that diphtheritic membrane could not be detached without tearing the underlying surface, while the croupous could be removed without injury. $\mathrm{He}$, however, surrendered this distinction after closer observation.

Dr. McKenzie holds that it has been fully demonstrated that the difference in degree of adhesion of pharyngeal and laryngeal false membrane, is due to the difference in the structure of the parts upon which they exude.

So far as I am informed, microscopists have failed to differentiate. Dr. Wagner declares that his preparations of croupous and diphtheritic membrane "are very much alike." Rindfleisch admits that "the pathological processes are the same."

We now come to consider the supposed clinical difference and post mortem revelations.

Dr. Watson (see lectures as to membranous croup) says: "It is not contagious, but is sometimes found attacking more than one member of the same family at the same time, or in quick succession. The children of washerwomen are more particularly obnoxious to it, and the attack is sometimes preceded by sore throat " (an incident has recently occurred under my own observation, in which diphtheria was conveyed in the clothes to be washed to the family of the laundress).

Dr. Colsy, in his account of membranous croup, says: "It appears to be epidemic when the wind is from the east" ; and in his descriptions of post mortem revelations says: "The mucous membrane of the pharynx is sometimes covered with false membrane, at the same time that we find it in the larynx."

Dr. Wood (see Practice, Vol. I., p. 81 3 , article Pseudo-Membranous Croup) says, in his account of symptoms: "This disease may always be suspected when the voice cannot be raised above a whisper, with wheezing inspirations, and especially when examination reveals patches upon the fauces, or a contimuous coating of fibrinous exudation on the soft palate, half arches, or pharynx."

"Troupian, in his lecture, "Diphtheria," says: "Diphtheria shows a marked preference for the pharynx, the air passages, and of them, particularly the larynx, constituting the affections commonly known as membranous sore throat, or malignant sore throat, formerly designated gangrenous sore throat, and suffocative sore throat, now more particularly called croup, in which the larynx is the chief seat of the disease. Of all its forms, pharyngeal is by far the most common."

Then follows the history of a case of croup occurring in the Hotel Dieu, under the following circumstances: Six days after the admission of a mother and her child into a ward, where there was a woman with membranous sore throat and a child with croup, the mother was found to have the right tonsil and uvula coated with false membrane and the cervical glands enlarged. Her child was attacked three days later. A whitish concretion was observed on a slightly abraded surface a: one of the commissures of the lips; the tonsils, as well as pillars and arch of the palate, presented nothing abnormal, not even redness. On the third day symptoms of croup presented them. selves; by the fifth this child was dead from suffocation. The autopsy revealed an absence of any deposit upon the tonsils or palate, but the larynx and trachea were invaded by faise membrane, which extended even to the most distant bronchial ramifications.

It is claimed by dualists that diphtheria has its local expression in the pharynx, occasionally spreading to the larynx, while croup is especially a disease of the larynx and trachea.

I have good authority for the statement that membranous croup originates in the larynx or trachea in about ten or twelve per cent. of cases, but if it had its origin in one of these localities in every case, unity could still be successfully maintained. In a constitutional disease local expression cannot make a specific difference.

Rheumatism, whether expressed in muscle or joint, is rheumatism still. Cancer is no less cancer with its local expression in the larynx, than if the pharynx be the site selected. It is true, if this site be selected the cervical glands sympathize at once, because of the intimate anatomical relations of the parts, and this fact obtains also in pharyngeal diphtheria, and admits of the same explanation.

But dualists urge that croup is a sthenic and diptheria, an asthenic disease. In reply, while few of the profession now resort to the lancet in membranous croup, but on the contrary distinguished dualist authorities recommend the supporting plan in which alcohol is specially included, Dr. Simons, of Paris, reports fifty-three cases of diphtheria treated by venesection in 1878 , without the loss of a case. All clinical experience attests a wide range in type of all the Zymoses, from collapse to the highest excitement. I believe laryngeal is generally more sthenic than pharyngeal diptheria, which fact has been ascribed to different anatomical relations of the local expression, but few authorities now regard membranous croup a sthenic disease.

Tosum up, unity is established by the history. Theold writers evidently describe diphtheria under the varied nomenclature of "croups, membranous croups, cynanche trachialis," etc. By the ætiology, none of us suppose that the children of London washerwomen were so prone to croup because of the dampness produced in their houses by washing, as Watson thought, but the contagion was brought in the clothing to be washed.

Pathologically, microscopically, and clinically, the membranous exudation has been shown to be the same, modified only by difference in site.

Clinically, for myself I will say, that if you will show me through the intervention of the laryngoscope false membranes in the larynx (eliminating Herpes and perhaps Minguett) or the expectorated membrane separated from the mucous by water, I will not hesitate to say that you have a case of blood-poisoniug, now known as diphtheria, although there were no deposits in the pharynx. I have recently treated and lost a case. 